MATHEMATICS OF COMPUTATION

Volume 69, Number 229, Pages 141-158

S 0025-5718(99)01205-3

Article electronically published on August 25, 1999

\title{
ULTRACONVERGENCE OF THE PATCH RECOVERY TECHNIQUE II
}

\author{
ZHIMIN ZHANG
}

\begin{abstract}
The ultraconvergence property of a gradient recovery technique proposed by Zienkiewicz and Zhu is analyzed for the Laplace equation in the two dimensional setting. Under the assumption that the pollution effect is not present or is properly controlled, it is shown that the convergence rate of the recovered gradient at an interior node is two orders higher than the optimal global convergence rate when even-order finite element spaces and local uniform rectangular meshes are used.
\end{abstract}

\section{INTRODUCTION}

In a previous work [6], the ultraconvergence property (i.e., two orders higher than the optimal global convergence rate) of the Zienkiewicz-Zhu patch recovery technique was justified for a class of two-point boundary value problems. The current work is devoted to the theoretical justification of the ultraconvergence phenomenon in the two dimensional setting. The main difficulty in the higher-dimensional situation is that when the domain has a nonsmooth boundary, the solution may have corner singularity, and consequently, the finite element approximation may suffer from "pollution effect", which will result in the failure of the recovery procedure. There have been many techniques to treat the pollution effect caused by domain singularity, and perhaps the most popular one is local mesh refinement. In this paper, we assume that a certain method is applied and the pollution effect is under control. Therefore we can concentrate on the local recovery. To further simplify the matter, we use the Laplace equation as the model problem. Then it can be shown that the patch recovery procedure results in ultraconvergence gradient recovery at an interior node when local uniform rectangular meshes and even-order elements are used.

\section{The PATCh RECOVERY TECHNique}

Consider the Dirichlet problem on a bounded domain $\Omega \subset \mathbb{R}^{2}$ with a piecewise smooth boundary $\partial \Omega$ :

$$
-\Delta u=f \quad \text { in } \Omega,\left.\quad u\right|_{\partial \Omega}=0 .
$$

We assume that $f$ is sufficiently smooth for our analysis.

Received by the editor August 7, 1996.

1991 Mathematics Subject Classification. Primary 65N30; Secondary 65N15.

This work was supported in part under NSF Grants No. DMS-9626193, No. DMS-9622690 and No. INT-9605050. 
The weak formulation of $(2.1)$ is to find $u \in H_{0}^{1}(\Omega)$ such that

$$
\int_{\Omega} \nabla u \nabla v d x d y=\int_{\Omega} f v d x d y, \quad \forall v \in H_{0}^{1}(\Omega) .
$$

Let $\mathcal{T}_{h}$ be a sequence of subdivisions of $\bar{\Omega}$. An element of $\mathcal{T}_{h}$ can be either a triangle or a quadrilateral. But we assume that rectangular elements are used on an interior region away from the boundary $\partial \Omega$. It is on this region that the patch recovery technique will be applied and analyzed. The finite element space $S_{h}(\Omega)$ is defined as usual, and it contains continuous piecewise polynomials of degree not greater than $r$.

Now we define local finite element spaces for rectangular elements. Given a rectangular element $K$, let $F_{K}$ be the linear mapping that maps the reference element $\widehat{K}=[-1,1] \times[-1,1]$ onto $K$. We then denote $\hat{v}=v \circ F_{K}$ for function $v$ on element $K$.

We assume on $\widehat{K}$, that the polynomial basis $\mathcal{P}_{r}$ contains $P(r)$ and is contained in $Q(r)$, where $P(r)$ denotes the class of polynomials which are of degree $r$, and $Q(r)$, the class of polynomials of degree $r$ in each variable separately. In other words, any element $p \in P(r)$ has the form

$$
p(\xi, \eta)=\sum_{i+j=0}^{r} c_{i j} \xi^{i} \eta^{j},
$$

whereas any $q \in Q(r)$ will be of the form

$$
q(\xi, \eta)=\sum_{i=0}^{r} \sum_{j=0}^{r} c_{i j} \xi^{i} \eta^{j}
$$

Some popular choices of polynomial basis $\mathcal{P}_{r}$ for rectangular (quadrilateral) finite elements are:

(I) Serendipity family: $P(r) \cup\left\{\xi^{r} \eta, \xi \eta^{r}\right\}$.

(II) Intermediate family of the first type:

$$
P(r+1) \cap Q(r)=P(r) \cup\left\{\xi^{r} \eta, \xi^{r-1} \eta^{2}, \ldots, \xi \eta^{r}\right\}=P(r+1) \backslash\left\{\xi^{r+1}, \eta^{r+1}\right\} .
$$

(III) Intermediate family of the second type:

$$
\begin{aligned}
P(r+2) \cap Q(r) & =P(r) \cup\left\{\xi^{r} \eta, \xi^{r-1} \eta^{2}, \ldots, \xi \eta^{r} ; \xi^{r} \eta^{2}, \xi^{r-1} \eta^{3}, \ldots, \xi^{2} \eta^{r}\right\} \\
& =P(r+2) \backslash\left\{\xi^{r+1}, \eta^{r+1} ; \xi^{r+2}, \xi^{r+1} \eta, \xi \eta^{r+1}, \eta^{r+2}\right\} .
\end{aligned}
$$

(IV) Tensor-product elements: $Q(r)$.

Note that

$$
P(r) \cup\left\{\xi^{r} \eta, \xi \eta^{r}\right\} \subset P(r+1) \cap Q(r) \subset P(r+2) \cap Q(r) \subset Q(r) .
$$

When $r=1$, they are all the same; when $r=2$, (I), (II) are the same, and (III), (IV) are the same; when $r \geq 3$, they are all different.

The finite element solution of (2.2) is to find $u_{h} \in S_{h}^{0}(\Omega)=S_{h}(\Omega) \cap H_{0}^{1}(\Omega)$ such that

$$
\int_{\Omega} \nabla u_{h} \nabla v d x d y=\int_{\Omega} f v d x d y, \quad \forall v \in S_{h}^{0}(\Omega)
$$

Since all of the four bases above contain $P(r)$, the optimal convergence rate for the gradient of the finite element solution is of order $O\left(h^{r}\right)$. We shall show that the 
patch recovery procedure is able to achieve $O\left(h^{r+1}\right)$ on an interior element patch and $O\left(h^{r+2}\right)$ at an interior nodal point.

In order to define the recovered gradient, we introduce the Gauss points and the Lobatto points.

Let $L_{r}(x)$ be the Legendre polynomial of degree $r$ on $[-1,1]$. It is well known that $L_{r}(x)$ has $r$ zeros and $L_{r}^{\prime}(x)$ has $r-1$ zeros in $(-1,1)$. Denote by $g_{1}^{(r)}, \ldots, g_{r}^{(r)}$ the zeros of $L_{r}(x)$, and by $l_{1}^{(r)}, \ldots, l_{r-1}^{(r)}$ the zeros of $L_{r}^{\prime}(x)$ with $l_{0}^{(r)}=-1, l_{r}^{(r)}=1$. Then $g_{j}^{(r)}, j=1, \ldots, r$, are called the Gauss points of order $r$, and $l_{j}^{(r)}, j=0,1, \ldots, r$, the Lobatto points of order $r$.

The Gauss and Lobatto points on a rectangle are defined as the tensor product of affine transformations of $g_{j}^{(r)}$ and $l_{j}^{(r)}$ of the respective rectangle. To be more precise, for a rectangle centered at $(\bar{x}, \bar{y})$ with length $h$ and height $\hbar$, the Gauss and Lobatto points are

$$
\begin{aligned}
& G_{i j}^{(r)}=\left(\bar{x}+g_{i}^{(r)} h / 2, \bar{y}+g_{j}^{(r)} \hbar / 2\right), \quad i, j=1, \ldots, r, \\
& L_{i j}^{(r)}=\left(\bar{x}+l_{i}^{(r)} h / 2, \bar{y}+l_{j}^{(r)} \hbar / 2\right), \quad i, j=0,1, \ldots, r .
\end{aligned}
$$

The Gauss and Lobatto points on an arbitrary quadrilateral can be defined through a bilinear mapping. In this paper, we focus our analysis on the element patch that contains four rectangles which share a common node.

In general, $\nabla u_{h}$ is a piecewise polynomial vector field and is discontinuous across element edges. The recovered gradient by the patch recovery is a continuous piecewise polynomial vector field, $R \nabla u_{h} \in S_{h}(\Omega) \times S_{h}(\Omega)$, which is uniquely determined by its values at the Lobatto points. The values of the recovered gradient at the Lobatto points are obtained by the following least squares fitting procedure. On an element patch $D_{0}$ (it contains four rectangles that share a common node, as we mentioned earlier), consider a polynomial in $\mathcal{P}$ with $P(r) \subset \mathcal{P} \subset Q(r)$ (for the serendipity family and the intermediate family of the first type), or $P(r+1) \cap Q(r) \subset$ $\mathcal{P} \subset Q(r)$ (for the tensor product element and the intermediate family of the second type),

$$
p_{r}^{*}(x, y)=\left(1, x, y, x^{2}, x y, y^{2}, \ldots\right) \mathbf{a} .
$$

The vector $\mathbf{a}=\left(a_{0}, a_{1}, \ldots, a_{m}\right)^{T}$ is computed by fitting, in the least squares sense, $\frac{\partial u_{h}}{\partial x}$ (or $\left.\frac{\partial u_{h}}{\partial y}\right)$ at $4(r \times r)$ Gauss points. Then the values of $R \frac{\partial u_{h}}{\partial x}$ at the Lobatto points are the values of $p_{r}^{*}$ at the same points. Note that adjacent element patches overlap. If different patches result in different recoveries at a Lobatto point in an overlapped region, an averaging is applied (see [8] for more details).

\section{UltraconVERGENCE ANALYSis}

Define

$$
\phi_{k}(\xi)=\sqrt{\frac{2 k-1}{2}} \int_{-1}^{\xi} L_{k-1}(t) d t
$$

and recall that $L_{k-1}$ is the Legendre polynomial of degree $k-1$. Then we have

$$
\phi_{k}(-1)=\phi_{k}(1)=0, \quad \int_{-1}^{1} \phi_{k}^{\prime}(\xi) d \xi=0, \quad \int_{-1}^{1} \phi_{k}^{\prime}(\xi) \phi_{l}^{\prime}(\xi) d \xi=0, \quad k \neq l .
$$


Here we adopt a set of hierarchic shape functions used in practical engineering code (cf., e.g., [4, section 6.1]). They are defined on the reference element and organized into three categories:

1) Nodal shape functions. There are 4 of them:

$$
\begin{array}{lll}
\text { Node } 1 & N_{1}(\xi, \eta)=\frac{1}{4}(1-\xi)(1-\eta) ; \\
\text { Node } 2 & N_{2}(\xi, \eta)=\frac{1}{4}(1+\xi)(1-\eta) ; \\
\text { Node } 3 & N_{3}(\xi, \eta)=\frac{1}{4}(1+\xi)(1+\eta) ; \\
\text { Node } 4 & N_{4}(\xi, \eta)=\frac{1}{4}(1-\xi)(1+\eta) .
\end{array}
$$

2) Side modes. There are $4(r-1)(r \geq 2)$ of them and $r-1$ on each side. For Side 1 (between Node 1 and Node 2) we have

$$
N_{1 j}(\xi, \eta)=\frac{1}{2}(1-\eta) \phi_{j}(\xi), \quad j=2, \ldots, r ;
$$

and for Side 2 (between Node 2 and Node 3) we have

$$
N_{2 j}(\xi, \eta)=\frac{1}{2}(1+\xi) \phi_{j}(\eta), \quad j=2, \ldots, r .
$$

The modes on the other two sides are defined accordingly.

3) Internal modes.

$$
\begin{aligned}
N_{01}(\xi, \eta) & =\phi_{2}(\xi) \phi_{2}(\eta), \\
N_{02}(\xi, \eta) & =\phi_{3}(\xi) \phi_{2}(\eta), \\
N_{03}(\xi, \eta) & =\phi_{2}(\xi) \phi_{3}(\eta), \\
N_{04}(\xi, \eta) & =\phi_{4}(\xi) \phi_{2}(\eta), \\
N_{05}(\xi, \eta) & =\phi_{3}(\xi) \phi_{3}(\eta), \\
N_{06}(\xi, \eta) & =\phi_{2}(\xi) \phi_{4}(\eta), \\
\text { etc. } &
\end{aligned}
$$

The following is a list of the number of internal modes for rectangular finite element families mentioned in the previous section.

$$
\begin{aligned}
\mathcal{P}_{r} & \text { Number of internal modes } \\
P(r) \cup\left\{\xi^{r} \eta, \xi \eta^{r}\right\} & (r-2)(r-3) / 2 \quad r \geq 4, \\
P(r+1) \cap Q(r) & (r-1)(r-2) / 2 \quad r \geq 3, \\
P(r+2) \cap Q(r) & r(r-1) / 2 \quad r \geq 2, \\
Q(r) & (r-1)^{2} \quad r \geq 2 .
\end{aligned}
$$

Based on this set of hierarchic shape functions, we shall define a special interpolation $u_{I}$ for a given function $u$. We will see that $u_{I}$ has some surprisingly nice superconvergent properties that the traditional interpolating functions do not have. These properties allow $\nabla u_{I}$ to be used as a vehicle in proving the superconvergence of the recovered finite element gradient $R \nabla u_{h}$. 


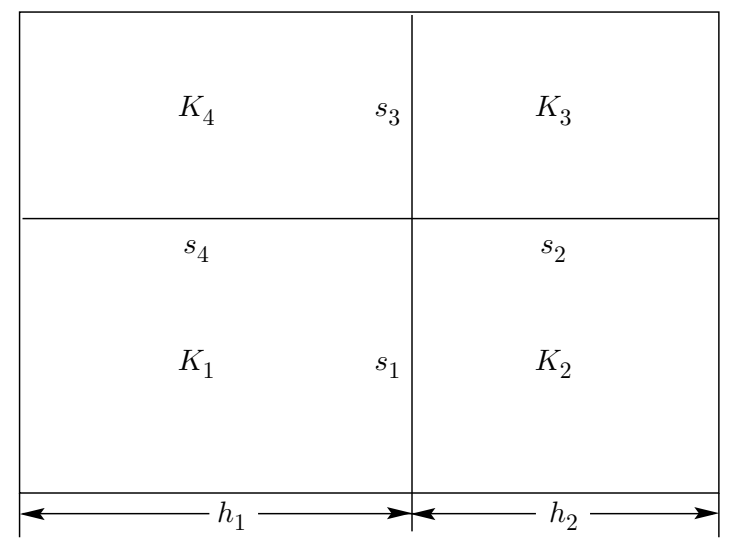

FiguRE 1

Given a smooth function $u(x, y)$, we consider $\hat{u}(\xi, \eta)=u(x(\xi, \eta), y(\xi, \eta))$ on the reference element $[-1,1]^{2}$ and define $u_{I}(x, y)$ with $\hat{u}_{I} \in \mathcal{P}_{r}$, such that

$$
\hat{u}_{I}(\xi, \eta)=\sum_{i=1}^{4} u_{i} N_{i}(\xi, \eta)+\sum_{i=1}^{4} \sum_{j=2}^{r} u_{j}^{(i)} N_{i j}(\xi, \eta)+\sum_{j} u_{j}^{(0)} N_{0 j}(\xi, \eta),
$$

with the coefficients decided by (i) $\hat{u}_{I}( \pm 1, \pm 1)=\hat{u}( \pm 1, \pm 1)$; (ii)

$$
\int_{\partial \widehat{K}} \frac{\partial}{\partial s}\left(\hat{u}-\hat{u}_{I}\right) \frac{\partial \hat{v}}{\partial s} d s=0
$$

for any side mode $\hat{v}$; and (iii)

$$
\int_{\widehat{K}} \nabla\left(\hat{u}-\hat{u}_{I}\right) \nabla \hat{v} d \xi d \eta=0,
$$

for any internal mode $\hat{v}$. Recall the structure of the hierarchic shape functions. It is straightforward to verify that the definition above is meaningful and decides $u_{I}$ uniquely. Note that (i) and (ii) guarantee that $u_{I}$ is continuous across elements.

The relationship of the special interpolation $u_{I}$ with the traditional interpolation can be revealed by the following observation:

If $\hat{u} \in Q(r+1)$, and $\mathcal{P}_{r}=Q(r)$, then $\hat{u}_{I}$ is the Lagrange interpolation of $\hat{u}$ at the $(r+1) \times(r+1)$ Lobatto points.

Remarkable recovery properties of $u_{I}$ under $\boldsymbol{R}$ can be seen from the following key lemma.

Lemma 3.1. Let an element patch $D_{0}$ contain four rectangles that share a common node. (a) If $u \in P(r+1)\left(D_{0}\right)(r \geq 1)$ and the local finite element space $\mathcal{P}_{r}$ on $D_{0}$ contains the intermediate family of the first type, then $R \nabla u_{I}=\nabla u$ on $D_{0}$; (b) if the four rectangles in $D_{0}$ are uniform, $u \in P(2 s+2)\left(D_{0}\right)(s \geq 1)$, and the local finite element space $\mathcal{P}_{2 s}$ on $D_{0}$ contains the intermediate family of the second type, then $\boldsymbol{R} \nabla u_{I}=\nabla u$ at the center of $D_{0}$.

Proof. Without loss of generality, we assume that $D_{0}$ is centered at the origin. The four rectangles are denoted as $K_{i}, i=1,2,3,4$ (see Figure 1). 
(a) Since the finite element space has all terms in $P(r+1)\left(D_{0}\right) \cap Q(r)\left(D_{0}\right)$, we only need to prove the statement for

$$
u \in P(r+1)\left(D_{0}\right) \backslash Q(r)\left(D_{0}\right)=\left\{x^{r+1}, y^{r+1}\right\} .
$$

Let $u=x^{r+1}$. It can be verified from the definition of the special interpolation $u_{I}$ that

$$
u-u_{I}= \begin{cases}a \phi_{r+1}\left(2 x / h_{1}+1\right), & \text { in } K_{1} \text { and } K_{4}, \\ a \phi_{r+1}\left(2 x / h_{2}-1\right), & \text { in } K_{2} \text { and } K_{3},\end{cases}
$$

where $h_{i}(i=1,2)$ are the lengths of the rectangles.

Recall $\phi_{r+1}^{\prime}(\xi)=\sqrt{(2 r+1) / 2} L_{r}(\xi)$, and we see that $\frac{\partial}{\partial x}\left(u-u_{I}\right)=0$ along $r$ Gaussian lines in each $K_{i}, i=1,2,3,4$. By the least squares fitting procedure, $R \frac{\partial u_{I}}{\partial x}$ is a polynomial of degree $r$ in $x$ and equals $\frac{\partial u}{\partial x}$ at $2 r$ distinct points in $D_{0}$. Hence $R \frac{\partial u_{I}}{\partial x}=\frac{\partial u}{\partial x}$ on $D_{0}$, and therefore $R \nabla u_{I}=\nabla u$ on $D_{0}$ since it is trivial to verify $\frac{\partial u_{I}}{\partial y}=0=\frac{\partial u}{\partial y}$.

The case $u=y^{r+1}$ can be proved similarly.

(b) Since the finite element space has all terms in $P(2 s+2)\left(D_{0}\right) \cap Q(2 s)\left(D_{0}\right)$, we only need to prove the statement for

$u \in P(2 s+2)\left(D_{0}\right) \backslash Q(2 s)\left(D_{0}\right)=\left\{x^{2 s+1}, y^{2 s+1} ; x^{2 s+2}, x^{2 s+1} y, x y^{2 s+1}, y^{2 s+2}\right\}$.

We discuss them separately.

(1) $u=x^{2 s+1}$ and $u=y^{2 s+1}$. The proof is the same as in (a) by setting $r=2 s$.

(2) $u=x^{2 s+1} y$. In this case,

$$
u-u_{I}= \begin{cases}a \phi_{2 s+1}(2 x / h+1) y / \hbar, & \text { in } K_{1} \text { and } K_{4}, \\ a \phi_{2 s+1}(2 x / h-1) y / \hbar, & \text { in } K_{2} \text { and } K_{3} .\end{cases}
$$

Note that the mesh is uniform. As in $(\mathrm{a}), \frac{\partial}{\partial x}\left(u-u_{I}\right)=0$ along $2 s$ Gaussian lines in each $K_{i}, i=1,2,3,4$; hence, $R \frac{\partial u_{I}}{\partial x}=\frac{\partial u}{\partial x}$ on $D_{0}$. Further,

$$
\frac{\partial}{\partial y}\left(u-u_{I}\right)= \begin{cases}a \phi_{2 s+1}(2 x / h+1) / \hbar, & \text { in } K_{1} \text { and } K_{4}, \\ a \phi_{2 s+1}(2 x / h-1) / \hbar, & \text { in } K_{2} \text { and } K_{3}\end{cases}
$$

Realizing that $\phi_{2 s+1}$ is an odd function, we see that

$$
\frac{\partial}{\partial y}\left(u-u_{I}\right)(x)=-\frac{\partial}{\partial y}\left(u-u_{I}\right)(-x) .
$$

Since $D_{0}$ has four uniform rectangles, the least squares fitting evaluated at the center of $D_{0}$ leads to the same coefficients for function values at the symmetric sampling points. Hence, cancellation occurs for all terms and consequently

$$
R \frac{\partial}{\partial y}\left(u-u_{I}\right)(0,0)=0 \text {. }
$$

Observe that

$$
R \frac{\partial u}{\partial y}(0,0)=0=\frac{\partial u}{\partial y}(0,0)
$$


Since $\frac{\partial u}{\partial y}=x^{2 s+1}$ is an odd function, we then have

$$
R \frac{\partial u_{I}}{\partial y}(0,0)=\frac{\partial u}{\partial y}(0,0)
$$

(3) $u=x^{2 s+2}$. Now

$$
u-u_{I}= \begin{cases}a \phi_{2 s+2}(2 x / h+1), & \text { in } K_{1} \text { and } K_{4}, \\ a \phi_{2 s+2}(2 x / h-1), & \text { in } K_{2} \text { and } K_{3} .\end{cases}
$$

Since $\phi_{2 s+2}^{\prime}(\xi)=\sqrt{(4 s+3) / 2} L_{2 s+1}(\xi)$ is an odd function, the least squares fitting evaluated at $(0,0)$ results in cancellation of all terms in $K_{1} \cup K_{2}$ and $K_{3} \cup K_{4}$ as explained in (2). Hence

$$
R \frac{\partial}{\partial x}\left(u-u_{I}\right)(0,0)=0
$$

Further, $\frac{\partial u}{\partial x}=(2 s+2) x^{2 s+1}$ is an odd function, $R \frac{\partial u}{\partial x}(0,0)=0=\frac{\partial u}{\partial x}(0,0)$. Hence

$$
R \frac{\partial u_{I}}{\partial x}(0,0)=\frac{\partial u}{\partial x}(0,0)
$$

Again, it is trivial to verify that $\frac{\partial u_{I}}{\partial y}=0=\frac{\partial u}{\partial y}$.

The analysis for terms $y^{2 s+1}, x y^{2 s+1}$ and $y^{2 s+2}$ is similar. Summing up, we have proved for $u \in P(2 s+2)\left(D_{0}\right)$,

$$
R \nabla u_{I}(0,0)=\nabla u(0,0)
$$

Examining the argument for $u=x^{2 s+2}$ in the proof of Lemma $3.1(3)$, we see that in order to have the cancellation at the center of $D_{0}$, a simple averaging will do the work. To be more precise, we define an averaging nodal recovery operator by symmetrically picking up some points in the element patch, and setting

$$
\widetilde{\boldsymbol{R}} \nabla u_{h}(0,0)=\sum_{i} \alpha_{i}\left(\nabla u_{h}\left(\bar{x}_{i}, \bar{y}_{i}\right)+\nabla u_{h}\left(-\bar{x}_{i}, \bar{y}_{i}\right)+\nabla u_{h}\left(-\bar{x}_{i},-\bar{y}_{i}\right)+\nabla u_{h}\left(\bar{x}_{i},-\bar{y}_{i}\right)\right),
$$

where $0<\bar{x}_{i}<h, 0<\bar{y}_{i}<\hbar$, and $\sum_{i} \alpha_{i}=1 / 4$. For this averaging nodal recovery operator, we have

Corollary 3.1. Let an element patch $D_{0}$ contain four uniform rectangles that share a common node. If $u \in P(2 s)\left(D_{0}\right)(s \geq 1)$ and the local finite element space $\mathcal{P}_{2 s-1}$ on $D_{0}$ contains the intermediate family of the first type, then $\widetilde{R} \nabla u_{I}=\nabla u$ at the center of $D_{0}$.

Now we define

$$
\begin{aligned}
\|\nabla v\|_{L_{\infty}(D)} & =\max \left(\left\|\frac{\partial v}{\partial x}\right\|_{L_{\infty}(D)},\left\|\frac{\partial v}{\partial y}\right\|_{L_{\infty}(D)}\right) \\
|\nabla v(z)| & =\max \left(\left|\frac{\partial v}{\partial x}(z)\right|,\left|\frac{\partial v}{\partial y}(z)\right|\right) .
\end{aligned}
$$

A direct consequence of Lemma 3.1 and Corollary 3.1 is the following theorem (also see [7] Theorem 3.1 and the Corollary for a different proof). 
Theorem 3.1. Let the element patch $D_{0}$ contain four rectangles that share a common node $z_{0}$, and let $D_{h}$ contain sixteen rectangles with the interior four elements being $D_{0}$ (see Figure 3 ).

(a) If the local finite element space $\mathcal{P}_{r}(r \geq 1)$ on $D_{0}$ contains the intermediate family of the first type and $u \in W_{\infty}^{r+2}\left(D_{h}\right)$, then

$$
\left\|\nabla u-R \nabla u_{I}\right\|_{L_{\infty}\left(D_{0}\right)} \leq C h^{r+1}|u|_{W_{\infty}^{r+2}\left(D_{h}\right)} .
$$

(b) If the four rectangles in $D_{0}$ are uniform, the local finite element space $\mathcal{P}_{2 s}$ $(s \geq 1)$ on $D_{0}$ contains the intermediate family of the second type, and $u \in$ $W_{\infty}^{2 s+3}\left(D_{0}\right)$, then

$$
\left|\left(\nabla u-R \nabla u_{I}\right)\left(z_{0}\right)\right| \leq C h^{2 s+2}|u|_{W_{\infty}^{2 s+3}\left(D_{0}\right)} .
$$

(c) If the four rectangles in $D_{0}$ are uniform, the local finite element space $\mathcal{P}_{2 s-1}$ $(s \geq 1)$ on $D_{0}$ contains the intermediate family of the first type, and $u \in W_{\infty}^{2 s+1}\left(D_{0}\right)$, then

$$
\left|\left(\nabla u-\widetilde{R} \nabla u_{I}\right)\left(z_{0}\right)\right| \leq C h^{2 s}|u|_{W_{\infty}^{2 s+1}\left(D_{0}\right)} .
$$

The constant $C$ is independent of $h$ and $u$.

Remark 3.1. Note that $D_{h}$ appears in the right-hand side of (3.1), since the recovery on $D_{0}$ involves the Gauss points on $D_{h}$.

Remark 3.2. We see that $R \nabla u_{I}$ is superconvergent to $\nabla u$ on the entire element patch $D_{0}$ when the local finite element space contains the intermediate family of the first type; $\widetilde{R} \nabla u_{I}$ is superconvergent to $\nabla u$ at the center of $D_{0}$ when the local finite element space contains the intermediate family of the first type with an odd degree; and $R \nabla u_{I}$ is ultraconvergent to $\nabla u$ at the center of $D_{0}$ when the local finite element space contains the intermediate family of the second type with an even degree.

Although $u_{I}$ is not the finite element solution, we are able to show that it is "almost" the finite element solution in the sense of the Lemma 3.2.

Lemma 3.2. Let a rectangular domain $D \subset \Omega$ be subdivided into rectangles $K \subset$ $D$.

(a) If $u \in P(r+1)(K)(r \geq 1)$ and the local finite element space $\mathcal{P}_{r}$ on $D$ contains the intermediate family of the first type, then

$$
\int_{K} \nabla\left(u-u_{I}\right) \nabla v d x d y=0, \quad \forall v \in S_{h}^{0}(D) .
$$

(b) If two rectangles $K$ and $K^{\prime}$ that share a common horizontal/vertical side are uniform, $u \in P(r+2)\left(K \cup K^{\prime}\right)(r \geq 2)$, and the local finite element space $\mathcal{P}_{r}$ on $D$ contains the intermediate family of the second type, then

$$
\int_{K \cup K^{\prime}} \nabla\left(u-u_{I}\right) \nabla v d x d y=0, \quad \forall v \in S_{h}^{0}(D)
$$

where $v=0$ on the rest two horizontal/vertical sides (see Figure 2).

Proof. Again, we only need to prove the claim for $u$ not included in the local finite element space. 

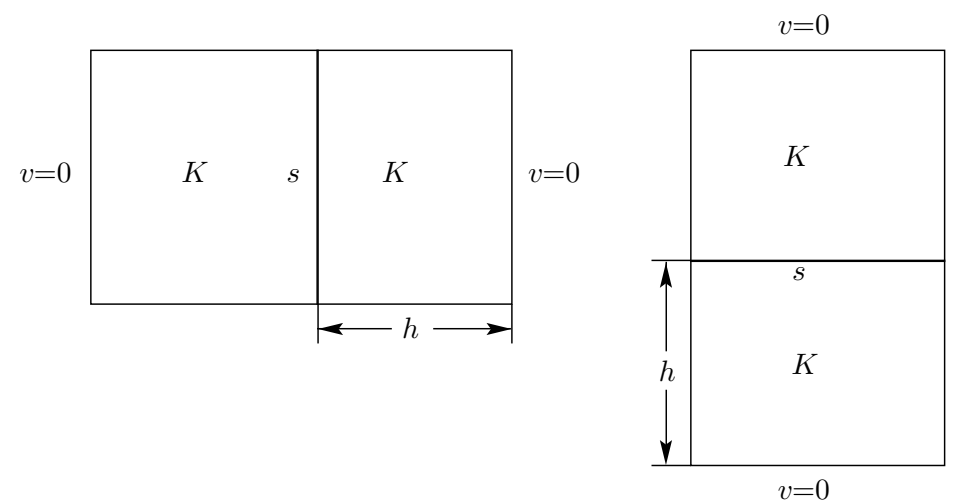

Figure 2

If $u=x^{k+1}, k=r, r+1$. From (a) of the proof for Lemma 3.1, we have on each rectangle $K \subset D$,

$$
\int_{K} \nabla\left(u-u_{I}\right) \nabla v d x d y=c \int_{-1}^{1} \int_{-1}^{1} L_{k}(\xi) \frac{\partial \hat{v}}{\partial \xi} d \xi d \eta=0, \quad \forall v \in S_{h}^{0}(D),
$$

since $\frac{\partial \hat{v}}{\partial \xi}$ is a polynomial of degree not exceeding $r-1$ in $\xi$. The argument for $u=y^{k+1}, k=r, r+1$ is the same. This proves (3.4) and special cases of (3.5) when $u=x^{r+2}, y^{r+2}, x^{r+1}, y^{r+1}$.

Recall the definition of $u_{I}$, for any $K \subset D$,

$$
\int_{K} \nabla\left(u-u_{I}\right) \nabla v d x d y=0
$$

if $v$ is an internal mode. Hence it remains to prove (3.5) for $u=x^{r+1} y, x y^{r+1}$, and $v$ being side modes and node modes. In order to include all the possible choices, we consider an element patch $D_{0}$ centered at the origin that contains four uniform rectangles which share a common node. Denoting by $s_{i}, i=1,2,3,4$, four sides between $K_{1}$ and $K_{2}, K_{2}$ and $K_{3}, K_{3}$ and $K_{4}, K_{4}$ and $K_{1}$, respectively (Figure 1 ). Let $v_{i, j}$ be the side mode on $s_{i}, j=2, \ldots, r$. Then

$$
\begin{aligned}
& v_{1, j}= \begin{cases}(1+x / h) \phi_{j}(2 y / \hbar+1), & \text { in } K_{1}, \\
(1-x / h) \phi_{j}(2 y / \hbar+1), & \text { in } K_{2} ;\end{cases} \\
& v_{2, j}= \begin{cases}(1+y / \hbar) \phi_{j}(2 x / h-1), & \text { in } K_{2}, \\
(1-y / \hbar) \phi_{j}(2 x / h-1), & \text { in } K_{3} ;\end{cases} \\
& v_{3, j}= \begin{cases}(1-x / h) \phi_{j}(2 y / \hbar-1), & \text { in } K_{3}, \\
(1+x / h) \phi_{j}(2 y / \hbar-1), & \text { in } K_{4} ;\end{cases} \\
& v_{4, j}= \begin{cases}(1-y / \hbar) \phi_{j}(2 x / h+1), & \text { in } K_{4}, \\
(1+y / \hbar) \phi_{j}(2 x / h+1), & \text { in } K_{1} .\end{cases}
\end{aligned}
$$




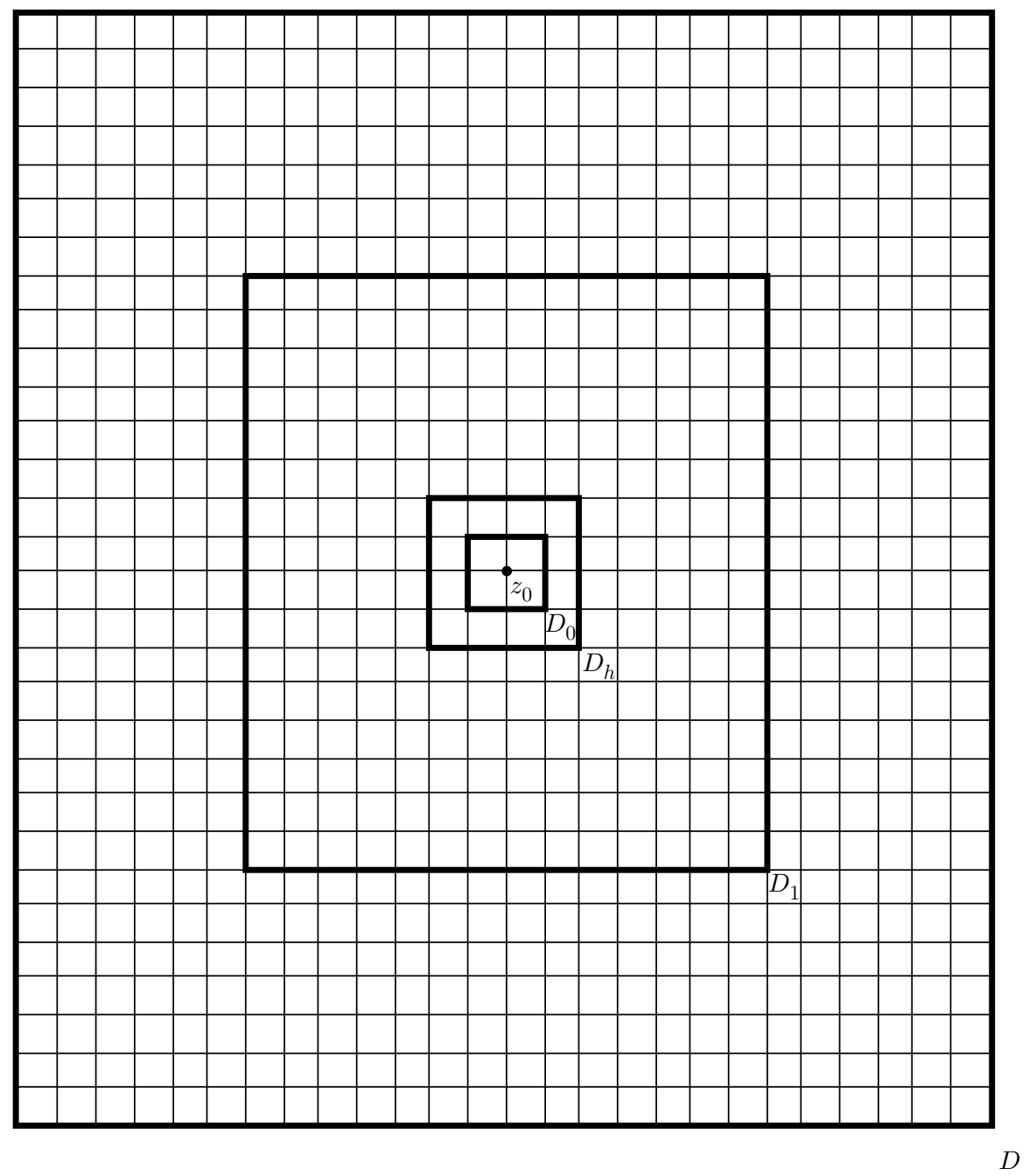

Figure 3

There is only one node mode whose support is in $D_{0}$ :

$$
v= \begin{cases}(1+x / h)(1+y / \hbar), & \text { in } K_{1}, \\ (1-x / h)(1+y / \hbar), & \text { in } K_{2}, \\ (1-x / h)(1-y / \hbar), & \text { in } K_{3}, \\ (1+x / h)(1-y / \hbar), & \text { in } K_{4} .\end{cases}
$$

Recall from the proof of Lemma 3.1 (b), if $u=x^{r+1} y$, then

$$
u-u_{I}= \begin{cases}a \phi_{r+1}(2 x / h+1) y / \hbar, & \text { in } K_{1} \text { and } K_{4}, \\ a \phi_{r+1}(2 x / h-1) y / \hbar, & \text { in } K_{2} \text { and } K_{3} .\end{cases}
$$


Hence

$$
\begin{aligned}
\int_{K_{1} \cup K_{2}} & \nabla\left(u-u_{I}\right) \nabla v_{1, j} d x d y \\
= & \frac{2 a}{h^{2}} \int_{K_{1}} \phi_{r+1}^{\prime}\left(\frac{2 x}{h}+1\right) \frac{y}{\hbar} \phi_{j}\left(\frac{2 y}{\hbar}+1\right) d x d y \\
& +\frac{2 a}{\hbar^{2}} \int_{K_{1}} \phi_{r+1}\left(\frac{2 x}{h}+1\right)\left(1+\frac{x}{h}\right) \phi_{j}^{\prime}\left(\frac{2 y}{\hbar}+1\right) d x d y \\
& -\frac{2 a}{h^{2}} \int_{K_{2}} \phi_{r+1}^{\prime}\left(\frac{2 x}{h}-1\right) \frac{y}{\hbar} \phi_{j}\left(\frac{2 y}{\hbar}+1\right) d x d y \\
+ & \frac{2 a}{\hbar^{2}} \int_{K_{2}} \phi_{r+1}\left(\frac{2 x}{h}-1\right)\left(1-\frac{x}{h}\right) \phi_{j}^{\prime}\left(\frac{2 y}{\hbar}+1\right) d x d y=0
\end{aligned}
$$

since all four terms contain one of

$$
\int_{-1}^{1} \phi_{k}^{\prime}(\xi) d \xi=0, \quad k=2, \ldots, r+1
$$

If $u=x y^{r+1}$, then

$$
u-u_{I}= \begin{cases}a \phi_{r+1}(2 y / \hbar+1) x / h, & \text { in } K_{1} \text { and } K_{2}, \\ a \phi_{r+1}(2 y / \hbar-1) x / h, & \text { in } K_{3} \text { and } K_{4}\end{cases}
$$

Hence

$$
\begin{aligned}
\int_{K_{1} \cup K_{2}} & \nabla\left(u-u_{I}\right) \nabla v_{1, j} d x d y \\
= & \frac{a}{h^{2}} \int_{K_{1}} \phi_{r+1}\left(\frac{2 y}{\hbar}+1\right) \phi_{j}\left(\frac{2 y}{\hbar}+1\right) d x d y \\
& +\frac{4 a}{\hbar^{2}} \int_{K_{1}} \phi_{r+1}^{\prime}\left(\frac{2 y}{\hbar}+1\right) \frac{x}{h}\left(1+\frac{x}{h}\right) \phi_{j}^{\prime}\left(\frac{2 y}{\hbar}+1\right) d x d y \\
& -\frac{a}{h^{2}} \int_{K_{2}} \phi_{r+1}\left(\frac{2 y}{\hbar}+1\right) \phi_{j}\left(\frac{2 y}{\hbar}+1\right) d x d y \\
& -\frac{4 a}{\hbar^{2}} \int_{K_{2}} \phi_{r+1}^{\prime}\left(\frac{2 y}{\hbar}+1\right) \frac{x}{h}\left(1-\frac{x}{h}\right) \phi_{j}^{\prime}\left(\frac{2 y}{\hbar}+1\right) d x d y=0,
\end{aligned}
$$

since the first and the third terms are canceled, and the second and fourth terms both contain the term

$$
\int_{-1}^{1} \phi_{r+1}^{\prime}(\xi) \phi_{j}^{\prime}(\xi) d \xi=\int_{-1}^{1} L_{r}(\xi) L_{j-1}(\xi) d \xi=0, \quad j=2, \ldots, r
$$

The argument for the remaining three side modes is similar and, hence, mode. is omitted. This finishes the proof of (3.5) when $v$ is a side mode. 
Next we consider the node mode. When $u=x^{r+1} y$,

$$
\begin{aligned}
\int_{K_{1} \cup K_{4}} \nabla\left(u-u_{I}\right) \nabla v d x d y \\
=\frac{2 a}{h^{2}} \int_{K_{1}} \phi_{r+1}^{\prime}\left(\frac{2 x}{h}+1\right) \frac{y}{\hbar}\left(1+\frac{y}{\hbar}\right) d x d y+\frac{a}{\hbar^{2}} \int_{K_{1}} \phi_{r+1}\left(\frac{2 x}{h}+1\right)\left(1+\frac{x}{h}\right) d x d y \\
\quad+\frac{2 a}{h^{2}} \int_{K_{4}} \phi_{r+1}^{\prime}\left(\frac{2 x}{h}+1\right) \frac{y}{\hbar}\left(1-\frac{y}{\hbar}\right) d x d y-\frac{a}{\hbar^{2}} \int_{K_{4}} \phi_{r+1}\left(\frac{2 x}{h}+1\right)\left(1+\frac{x}{h}\right) d x d y \\
=0
\end{aligned}
$$

since the first and the third terms both contain

$$
\int_{-1}^{1} \phi_{r+1}^{\prime}(\xi) d \xi=0
$$

and the second and the fourth terms are canceled. Similarly, we can prove

$$
\int_{K_{2} \cup K_{3}} \nabla\left(u-u_{I}\right) \nabla v d x d y=0 .
$$

The proof of the integral on $K_{1} \cup K_{2}$ needs some more work.

$$
\begin{aligned}
\int_{K_{1} \cup K_{2}} & \nabla\left(u-u_{I}\right) \nabla v d x d y \\
= & \frac{2 a}{h^{2}} \int_{K_{1}} \phi_{r+1}^{\prime}\left(\frac{2 x}{h}+1\right) \frac{y}{\hbar}\left(1+\frac{y}{\hbar}\right) d x d y+\frac{a}{\hbar^{2}} \int_{K_{1}} \phi_{r+1}\left(\frac{2 x}{h}+1\right)\left(1+\frac{x}{h}\right) d x d y \\
& -\frac{2 a}{h^{2}} \int_{K_{2}} \phi_{r+1}^{\prime}\left(\frac{2 x}{h}-1\right) \frac{y}{\hbar}\left(1+\frac{y}{\hbar}\right) d x d y+\frac{a}{\hbar^{2}} \int_{K_{2}} \phi_{r+1}\left(\frac{2 x}{h}-1\right)\left(1-\frac{x}{h}\right) d x d y \\
= & 0 .
\end{aligned}
$$

The first and the third terms are zeros. The cancellation of the second and the fourth terms can be seen from the following.

$$
\begin{gathered}
\int_{-h}^{0} \phi_{r+1}\left(\frac{2 x}{h}+1\right)\left(1+\frac{x}{h}\right) d x+\int_{0}^{h} \phi_{r+1}\left(\frac{2 x}{h}-1\right)\left(1-\frac{x}{h}\right) d x \\
=\int_{0}^{1}\left[\phi_{r+1}\left(1-\frac{2 x}{h}\right)+\phi_{r+1}\left(\frac{2 x}{h}+1\right)\right]\left(1-\frac{x}{h}\right) d x=0
\end{gathered}
$$

since when $r$ is an even number,

$$
\phi_{r+1}\left(1-\frac{2 x}{h}\right)+\phi_{r+1}\left(\frac{2 x}{h}+1\right)=0 ;
$$

and when $r$ is an odd number, it must be $r \geq 3$. Then it can be verified that $\phi_{r+1}$ is orthogonal to any linear function from its definition. Similarly, we can prove

$$
\int_{K_{3} \cup K_{4}} \nabla\left(u-u_{I}\right) \nabla v d x d y=0 .
$$

The argument for $u=x y^{r+1}$ is similar. Hence we have proved (3.5) when $v$ is a nodal shape function. This completes the proof of the lemma.

Theorem 3.2. Let the support of $u$ be contained in a rectangular domain $D$ which is subdivided into rectangles. 
(a) If the finite element space $\mathcal{P}_{r}(r \geq 1)$ on $D$ contains the intermediate family of the first type and $u \in W_{\infty}^{r+2}(D)$, then

$$
\left|\int_{D} \nabla\left(u-u_{I}\right) \nabla v d x d y\right| \leq C h^{r+1}|u|_{W_{\infty}^{r+2}(D)}|v|_{W_{1}^{1}(D)}, \quad \forall v \in S_{h}^{0}(D) .
$$

(b) If the finite element space $\mathcal{P}_{r}(r \geq 2)$ on $D$ contains the intermediate family of the second type, the rectangular subdivision is uniform, and $u \in W_{\infty}^{r+3}(D)$, then

$$
\left|\int_{D} \nabla\left(u-u_{I}\right) \nabla v d x d y\right| \leq C h^{r+2}|u|_{W_{\infty}^{r+3}(D)}|v|_{W_{1}^{1}(D)}, \quad \forall v \in S_{h}^{0}(D) .
$$

Here $C$ is a constant depending only on $r$ and the ratio of the horizontal and vertical sides of $K \subset D$.

Proof. (a) We decompose $D$ into elements $\cup_{K \subset D} K$, and write

$$
\int_{D} \nabla\left(u-u_{I}\right) \nabla v d x d y=\sum_{K \subset D} \int_{K} \nabla\left(u-u_{I}\right) \nabla v d x d y .
$$

Recall that the local finite element space $\mathcal{P}_{r}$ contains the intermediate family of the first type. Then we have, from Lemma 3.2 (a),

$$
\int_{K} \nabla\left(u-u_{I}\right) \nabla v d x d y=0, \quad \forall v \in S_{h}^{0}(D),
$$

whenever $u \in P(r+1)(K)(r \geq 1)$. By the Bramble-Hilbert lemma, there exists a constant $C$ depending only on $r$ and the ratio of the horizontal and the vertical sides of $K$, such that

$$
\left|\int_{K} \nabla\left(u-u_{I}\right) \nabla v d x d y\right| \leq C h^{r+1}|u|_{W_{\infty}^{r+2}(K)}|v|_{W_{1}^{1}(K)} .
$$

Therefore,

$$
\begin{aligned}
\left|\int_{D} \nabla\left(u-u_{I}\right) \nabla v d x d y\right| & \leq C h^{r+1}|u|_{W_{\infty}^{r+2}(D)} \sum_{K \subset D}|v|_{W_{1}^{1}(K)} \\
& =C h^{r+1}|u|_{W_{\infty}^{r+2}(D)}|v|_{W_{1}^{1}(D)} .
\end{aligned}
$$

(b) We denote by $\mathcal{S}$ the set of all interior sides in $D$, and write

$$
\int_{D} \nabla\left(u-u_{I}\right) \nabla v d x d y=\sum_{K \cap K^{\prime}=s \in \mathcal{S}} \int_{K \cup K^{\prime}} \nabla\left(u-u_{I}\right) \nabla v_{s} d x d y
$$

where $v_{s}=0$ on the two horizontal or vertical sides of $K$ and $K^{\prime}$ other than $s$ (see Figure 2). Recall that the local finite element space $\mathcal{P}_{r}$ contains the intermediate family of the second type, from Lemma 3.2 (b),

$$
\int_{K \cup K^{\prime}} \nabla\left(u-u_{I}\right) \nabla v_{s} d x d y=0, \quad K \cup K^{\prime}=s,
$$

whenever $u \in P(r+2)\left(K \cup K^{\prime}\right)(r \geq 2)$. By the Bramble-Hilbert lemma, there exists a constant $C_{1}(r)$ depending only on $r$ and the ratio of the horizontal and the vertical sides of $K$, such that

$$
\left|\int_{K \cup K^{\prime}} \nabla\left(u-u_{I}\right) \nabla v_{s} d x d y\right| \leq C_{1}(r) h^{r+2}|u|_{W_{\infty}^{r+3}\left(K \cup K^{\prime}\right)}\left|v_{s}\right|_{W_{1}^{1}\left(K \cup K^{\prime}\right)} .
$$

For the uniform mesh, the constant $C_{1}(r)$ is the same for any pair $K$ and $K^{\prime}$ that share a common side. Next, we show that there exists another constant $C_{2}(r)$ 
depending only on $r$ and the ratio of the horizontal and the vertical sides of $K$, such that

$$
\left|v_{s}\right|_{W_{1}^{1}\left(K \cup K^{\prime}\right)} \leq C_{2}(r)|v|_{W_{1}^{1}\left(K \cup K^{\prime}\right)},
$$

for any pair $K$ and $K^{\prime}$ that share a common side. Without loss of generality, we assume that $v$ is not a constant other than zero on any $K \subset D$. If so, we simply drop the zero term $\int_{K} \nabla\left(u-u_{I}\right) \nabla v d x d y$ from the sum

$$
\int_{D} \nabla\left(u-u_{I}\right) \nabla v d x d y=\sum_{K \subset D} \int_{K} \nabla\left(u-u_{I}\right) \nabla v d x d y .
$$

Having this in mind, if we set $|v|_{W_{1}^{1}\left(K \cup K^{\prime}\right)}=0$, the expansion of $v$ on the basis functions on $K \cup K^{\prime}$ has to be zero. Therefore, $v_{s}=0$ on $K \cup K^{\prime}$ due to the linear independence of the basis functions. For the finite dimensional (related to $r$ ) case, there is a constant $C_{2}(r)$ such that (3.8) holds. By the uniform mesh assumption, this $C_{2}(r)$ is universal for all element pairs. Its dependence on the ratio of the horizontal and the vertical sides (not the size of them) can be seen by mapping $K$ and $K^{\prime}$ onto the reference element and performing the standard argument. In light of (3.8), we have

$$
\begin{aligned}
\left|\int_{D} \nabla\left(u-u_{I}\right) \nabla v d x d y\right| & \leq C_{1}(r) C_{2}(r) h^{r+2}|u|_{W_{\infty}^{r+3}(D)} \sum_{K \cap K^{\prime}=s \in \mathcal{S}}|v|_{W_{1}^{1}\left(K \cup K^{\prime}\right)} \\
& \leq 4 C_{1}(r) C_{2}(r) h^{r+2}|u|_{W_{\infty}^{r+3}(D)}|v|_{W_{1}^{1}(D)} .
\end{aligned}
$$

Note that an element may repeat as many as four times since the overlapping.

We are interested in derivative recovery at an interior point or a small interior region (e.g., an element patch) which is away from the boundary $\partial \Omega$. We consider $D$, a fixed rectangular interior subdomain of $\Omega$. Suppose that a rectangular mesh refinement is used on $D$ that keeps its center $z_{0}$ as a node. Let $D_{0}$ be the element patch that contains four elements with the common node $z_{0}$, let $D_{h}$ be the element patch obtained by adding one more layer of elements to $D_{0}$, and let $D_{1}$ be a fixed rectangular domain whose boundary is about half way between the boundaries $\partial D_{0}$ and $\partial D$ (Figure 3 ). Then we have the following local analysis.

Theorem 3.3. Let $u$ be the solution of (2.2) and let $u_{h}$ be its finite element approximation defined by (2.3).

(a) If the finite element space $\mathcal{P}_{r}(r \geq 1)$ on $D$ contains the intermediate family of the first type and $u \in W_{\infty}^{r+2}(D)$, then

$$
\left|u_{I}-u_{h}\right|_{W_{\infty}^{1}\left(D_{h}\right)} \leq C h^{r+1}\left(|\ln h||u|_{W_{\infty}^{r+2}(D)}+h^{k}\|u\|_{H^{r+1}(D)}\right)+\left\|u-u_{h}\right\|_{H^{-k}(\Omega)},
$$

for a non-negative integer $k \leq r-1$.

(b) If the finite element space $\mathcal{P}_{r}(r \geq 2)$ on $D$ contains the intermediate family of the second type, the rectangular subdivision is uniform, and $u \in W_{\infty}^{r+3}(D)$, then

$$
\left|u_{I}-u_{h}\right|_{W_{\infty}^{1}\left(D_{0}\right)} \leq C h^{r+2}\left(\left|\ln h\left\|\left.u\right|_{W_{\infty}^{r+3}(D)}+h^{k-1}\right\| u \|_{H^{r+1}(D)}\right)+\left\|u-u_{h}\right\|_{H^{-k}(\Omega)},\right.
$$

for a positive integer $k \leq r-1$. Here $C$ is a constant independent of $u$ and $h$. 
Proof. We construct a cut-off function $\omega$ such that $\operatorname{Supp} \omega \subset D, \omega=1$ on $D_{1}$. We then decompose $u$ into $u=\tilde{u}+u^{\prime}$ with $\tilde{u}=u \omega$. For any $z \in D_{h}$, we define the discrete Green's function $G_{z}^{h} \in S_{h}^{0}(D)$ by

$$
\int_{D} \nabla G_{z}^{h} \nabla v d x d y=v(z), \quad \forall v \in S_{h}^{0}(D)
$$

Let $\partial_{z}$ denote any directional derivative with respect to $z$, then

$$
\int_{D} \nabla \partial_{z} G_{z}^{h} \nabla v d x d y=\partial_{z} v(z), \quad \forall v \in S_{h}^{0}(D) .
$$

It is known that $\left\|\partial_{z} G_{z}^{h}\right\|_{W_{1}^{1}(D)} \leq C|\ln h|$ (cf., [9, Theorem 3.14]) with the constant $C$ independent of $z$. Therefore,

$$
\partial_{z}\left(\tilde{u}_{I}-\tilde{u}_{h}\right)(z)=\int_{D} \nabla \partial_{z} G_{z}^{h} \nabla\left(\tilde{u}_{I}-\tilde{u}_{h}\right) d x d y=\int_{D} \nabla \partial_{z} G_{z}^{h} \nabla\left(\tilde{u}_{I}-\tilde{u}\right) d x d y .
$$

By Theorem 3.2, we have

$$
\left|\partial_{z}\left(\tilde{u}_{I}-\tilde{u}_{h}\right)(z)\right| \leq C h^{r+l}|u|_{W_{\infty}^{r+1+l}(D)}\left|\partial_{z} G_{z}^{h}\right|_{W_{1}^{1}(D)} \leq C h^{r+l}|\ln h||u|_{W_{\infty}^{r+1+l}(D)},
$$

where $l=1$ for (a) and $l=2$ for (b). Since $C$ is independent of $z$, we then have

$$
\left|\tilde{u}_{I}-\tilde{u}_{h}\right|_{W_{\infty}^{1}\left(D_{h}\right)} \leq C h^{r+l}|\ln h||u|_{W_{\infty}^{r+1+l}(D)} .
$$

Next, we consider $u_{I}^{\prime}-u_{h}^{\prime}$. Note that $u^{\prime}=0=u_{I}^{\prime}$ on $D_{1} \supset D_{h}$, we then have

$$
\left|u_{I}^{\prime}-u_{h}^{\prime}\right|_{W_{\infty}^{1}\left(D_{h}\right)}=\left|u_{h}^{\prime}\right|_{W_{\infty}^{1}\left(D_{h}\right)}=\left|u^{\prime}-u_{h}^{\prime}\right|_{W_{\infty}^{1}\left(D_{h}\right)} .
$$

Further,

$$
\int_{\Omega} \nabla\left(u^{\prime}-u_{h}^{\prime}\right) \nabla v d x d y=0, \quad \forall v \in S_{h}^{0}\left(D_{1}\right) .
$$

Hence, by [3, Theorem 1.2], we have for $1 \leq q \leq \infty$ and $k$ a nonnegative integer, that there exists a constant $C$ depending only on $q$ and $k$ such that

$$
\begin{aligned}
\left|u^{\prime}-u_{h}^{\prime}\right|_{W_{\infty}^{1}\left(D_{h}\right)} \leq & C \min _{\chi \in S_{h}}\left(\left|u^{\prime}-\chi^{\prime}\right|_{W_{\infty}^{1}\left(D_{1}\right)}+d^{-1}\left|u^{\prime}-\chi^{\prime}\right|_{L_{\infty}\left(D_{1}\right)}\right) \\
& +C d^{-1-k-2 / q}\left\|u^{\prime}-u_{h}^{\prime}\right\|_{W_{q}^{-k}\left(D_{1}\right)},
\end{aligned}
$$

where $d=\operatorname{dist}\left(\partial D_{h}, \partial D_{1}\right)$ which is a fixed number independent of $h$ by the construction of $D_{1}$. Since $u^{\prime}=0$ on $D_{1}$, only the last term on the right-hand side is left. By selecting $q=2$. We have

$$
\left|u^{\prime}-u_{h}^{\prime}\right|_{W_{\infty}^{1}\left(D_{h}\right)} \leq C\left\|u^{\prime}-u_{h}^{\prime}\right\|_{H^{-k}\left(D_{1}\right)} .
$$

Recall $u^{\prime}-u_{h}^{\prime}=u-\tilde{u}-\left(u_{h}-\tilde{u}_{h}\right)$, and notice that $\|v\|_{H^{-k}\left(D_{1}\right)} \leq\|v\|_{H^{-k}(D)} \leq$ $\|v\|_{H^{-k}(\Omega)}$. We then have

$$
\left|u^{\prime}-u_{h}^{\prime}\right|_{W_{\infty}^{1}\left(D_{h}\right)} \leq C\left(\left\|\tilde{u}-\tilde{u}_{h}\right\|_{H^{-k}(D)}+\left\|u-u_{h}\right\|_{H^{-k}(\Omega)}\right) .
$$

For the first term on the right-hand side with $k \leq r-1$, we apply the negative norm estimate for Dirichlet's problem on a rectangle $(D)$ given in $[1$, section 7 , Example 3] to have

$$
\left\|\tilde{u}-\tilde{u}_{h}\right\|_{H^{-k}(D)} \leq C h^{r+1+k}\|\tilde{u}\|_{H^{r+1}(D)} \leq C h^{r+1+k}\|u\|_{H^{r+1}(D)},
$$

since

$$
|u \omega|_{H^{r+1}(D)} \leq C \sum_{i=0}^{r+1}\left|D^{i} u D^{r+1-i} \omega\right|_{L_{2}(D)},
$$


and all derivatives of $\omega$ are independent of $h$. Hence

$$
\left|u_{I}^{\prime}-u_{h}^{\prime}\right|_{W_{\infty}^{1}\left(D_{h}\right)}=\left|u^{\prime}-u_{h}^{\prime}\right|_{W_{\infty}^{1}\left(D_{h}\right)} \leq C h^{r+1+k}\|u\|_{H^{r+1}(D)}+C\left\|u-u_{h}\right\|_{H^{-k}(\Omega)} .
$$

The conclusion follows by combining this with (3.11), and applying the triangle inequality.

In order to prove the main theorem of the paper, we assume that the pollution caused by the boundary singularity is properly controlled (for example, by local mesh refinement) in the sense that for some non-negative integer $k \leq r-1$,

$$
\left\|u-u_{h}\right\|_{H^{-k}(\Omega)} \leq C(f) h^{l},
$$

where $C(f)$ is a constant depending only on $f$ and $\Omega$, and $l=r+1,2 s+2$, or $2 s$, whenever it is necessary. For more details about negative norm estimates, see $[5$, section 6.3].

Theorem 3.4. Let $u$ be the solution of (2.2), let $u_{h}$ be its finite element approximation defined by (2.3), and let $z_{0} \in D_{0} \subset D_{h} \subset D_{1} \subset D \subset \Omega$ be defined as in Theorem 3.3. Assume that (3.12) is satisfied.

(a) If the local finite element space $\mathcal{P}_{r}(r \geq 1)$ on $D$ contains the intermediate family of the first type and $u \in W_{\infty}^{r+2}(D)$, then

$$
\left\|\nabla u-R \nabla u_{h}\right\|_{L_{\infty}\left(D_{0}\right)} \leq C h^{r+1}\left(\left|\ln h\left\|\left.u\right|_{W_{\infty}^{r+2}(D)}+h^{k}\right\| u \|_{H^{r+1}(D)}+C(f)\right),\right.
$$

for a non-negative integer $k \leq r-1$.

(b) If the local finite element space $\mathcal{P}_{2 s}(s \geq 1)$ on $D$ contains the intermediate family of the second type, the rectangular subdivision is uniform, and $u \in$ $W_{\infty}^{2 s+3}(D)$, then

$$
\left|\left(\nabla u-R \nabla u_{h}\right)\left(z_{0}\right)\right| \leq C h^{2 s+2}\left(|\ln h||u|_{W_{\infty}^{2 s+3}(D)}+h^{k-1}\|u\|_{H^{2 s+1}(D)}+C(f)\right),
$$

for a positive integer $k \leq 2 s-1$.

(c) If the local finite element space $\mathcal{P}_{2 s-1}(s \geq 1)$ on $D$ contains the intermediate family of the first type, the rectangular subdivision is uniform, and $u \in W_{\infty}^{2 s+1}(D)$. Then

$$
\left|\left(\nabla u-\widetilde{\boldsymbol{R}} \nabla u_{h}\right)\left(z_{0}\right)\right| \leq C h^{2 s}\left(|\ln h||u|_{W_{\infty}^{2 s+1}(D)}+h^{k}\|u\|_{H^{2 s}(D)}+C(f)\right),
$$

for a non-negative integer $k \leq 2 s-2$.

Proof. (a) By the triangle inequality

$$
\left\|\nabla u-\boldsymbol{R} \nabla u_{h}\right\|_{L_{\infty}\left(D_{0}\right)} \leq\left\|\nabla u-\boldsymbol{R} \nabla u_{I}\right\|_{L_{\infty}\left(D_{0}\right)}+\left\|\boldsymbol{R} \nabla\left(u_{I}-u_{h}\right)\right\|_{L_{\infty}\left(D_{0}\right)} .
$$

Observe that the recovery operator $\boldsymbol{R}$ is a bounded operator with an bound $C(r)$ depending only on $r$ and size ratios among elements in the patch. Then

$$
\left\|\boldsymbol{R} \nabla\left(u_{I}-u_{h}\right)\right\|_{L_{\infty}\left(D_{0}\right)} \leq C(r)\left\|\nabla\left(u_{I}-u_{h}\right)\right\|_{L_{\infty}\left(D_{h}\right)}=C(r)\left|u_{I}-u_{h}\right|_{W_{\infty}^{1}\left(D_{h}\right)} .
$$

Note that the recovered gradient on $D_{0}$ involves sampling Gauss points on $D_{h}$. Substituting (3.17) into (3.16), the conclusion follows by applying (3.1) and (3.9).

(b) Again, the triangle inequality gives

$$
\left|\left(\nabla u-R \nabla u_{h}\right)\left(z_{0}\right)\right| \leq\left|\left(\nabla u-R \nabla u_{I}\right)\left(z_{0}\right)\right|+\left|\boldsymbol{R} \nabla\left(u_{I}-u_{h}\right)\left(z_{0}\right)\right|,
$$

where

$$
\left|R \nabla\left(u_{I}-u_{h}\right)\left(z_{0}\right)\right| \leq C(s)\left\|\nabla\left(u_{I}-u_{h}\right)\right\|_{L_{\infty}\left(D_{0}\right)}=C(s)\left|u_{I}-u_{h}\right|_{W_{\infty}^{1}\left(D_{0}\right)} .
$$


Then we use (3.2) and (3.10) to achieve the desired result.

(c) The argument is the same as in (b) by using (3.3) and (3.9) with

$$
\left|\widetilde{\boldsymbol{R}} \nabla\left(u_{I}-u_{h}\right)\left(z_{0}\right)\right| \leq \sum_{i}\left|\alpha_{i}\right|\left|u_{I}-u_{h}\right|_{W_{\infty}^{1}\left(D_{0}\right)} .
$$

Applying Theorem 3.4 to the four rectangular finite element families I-IV introduced in Section 2, we summarize the results.

1) When $r=1$, all four families reduce to the same bilinear element. If the element center (Gauss point) is used as a sampling point, we will have superconvergence recovery on the whole element patch even when the rectangular mesh is nonuniform. But if arbitrary sampling points are selected symmetrically (one from each element), superconvergence recovery only occurs at the center of the patch that contains four uniform rectangles.

2) When $r=2$, I and II are the same - the quadratic serendipity element; III and IV are the same - the quadratic tensor-product element. For all cases, if the Gauss points (four in each element) are used as sampling points, superconvergence recovery is achieved on the whole element patch even for nonuniform rectangular meshes. Further, for the tensor product element, ultraconvergence recovery occurs at the center of the patch that contains four uniform rectangles.

3) When $r \geq 3$, for families II-IV, if the Gauss points ( $r^{2}$ in each element) are used as sampling points, superconvergence recovery is achieved on the whole element patch. But when $r$ is an odd number and the patch contains four uniform rectangles, a simple averaging of four symmetric points on the patch will result in superconvergence recovery at the center of the patch. For families III and IV, when $r$ is an even number and the Gauss points are used as sampling points, except superconvergence recovery on the whole patch, we have ultraconvergence recovery at the center of the patch that contains four uniform rectangles.

Remark 3.3. As a byproduct, Theorem 3.4 (c) explains the observation from numerical tests [8] that superconvergence recovery occurs at the element nodal point (patch center) with arbitrary sampling points (not Gauss points) for odd-order finite elements when uniform meshes are used (see [2] for additional superconvergence results in this respect involving local symmetry). However, our theory suggests the use of the Gauss points to achieve the best possible recovery.

\section{ACKNOWLEDGMENT}

The author would like to thank Professor Wahlbin for stimulating discussions on the topic of the interior estimate.

\section{REFERENCES}

1. J.A. Nitsche and A.H. Schatz, Interior estimates for Ritz-Galerkin methods, Math. Comp. $\mathbf{2 8}$ (1974), 937-958. MR 51:9525

2. A.H. Schatz, I.H. Sloan, and L.B. Wahlbin, Superconvergence in finite element methods and meshes that are locally symmetric with respect to a point, SIAM J. Numer. Anal. 33 (1996), 505-521. MR 98f:65112

3. A.H. Schatz and L.B. Wahlbin, Interior maximum-norm estimates for finite element methods. II, Math. Comp. 64 (1995), 907-928. MR 95j:65143

4. B. Szabó and I. Babuška, Finite Element Analysis, John Wiley \& Sons, New York, 1991. MR 93f: 73001

5. L.B. Wahlbin, Superconvergence in Galerkin Finite Element Methods, Lecture Notes in Mathematics, Vol. 1605, Springer, Berlin, 1995. MR 98j:65083 
6. Z. Zhang, Ultraconvergence of the patch recovery technique, Math. Comp. 65 (1996), 14311437. MR 97a:65068

7. Z. Zhang and H.D. Victory Jr., Mathematical analysis of Zienkiewicz-Zhu's derivative patch recovery technique, Numerical Methods for PDEs 12 (1996), 507-524. MR 98c:65191

8. O.C. Zienkiewicz and J.Z. Zhu, The superconvergence patch recovery and a posteriori error estimates. Part 1: The recovery technique, Internat. J. Numer. Meth. Eng. 33 (1992), 13311364.

9. Q.-D. Zhu and Q. Lin, Hyperconvergence Theory of Finite Elements, Hunan Science and Technology Publishing House, Changsha, P.R. China, 1989 (in Chinese). MR 93j:65191

Department of Mathematics, Texas Tech University, Lubbock, Texas 79409

E-mail address: zhang@ttmath.ttu.edu 